\title{
Comparison of the Virulence Factors and Analysis of Hypothetical Sequences of the Strains TIGR4, D39, G54 and R6 of Streptococcus Pneumoniae
}

\author{
R. Jothi, S. Parthasarathy* and K. Ganesan \\ Department of Bioinformatics, School of Life Sciences \\ Bharathidasan University, Tiruchirappalli 620 024, \\ Tamil Nadu, India \\ *Corresponding author : S. Parthasarathy, Department of Bioinformatics, \\ School of Life Sciences, Bharathidasan University, \\ Tiruchirappalli 620 024,Tamil Nadu, India, \\ Tel: +91 94435 33095; Fax: +91 431 2407045; E-mail: bdupartha@ gmail.com
}

Received October 21, 2008; Accepted November 19, 2008; Published December 26, 2008

Citation: Jothi R, Parthasarathy S, Ganesan K (2008) Comparison of the Virulence Factors and Analysis of Hypothetical Sequences of the Strains TIGR4, D39, G54 and R6 of Streptococcus Pneumoniae .JComput Sci Syst Biol 1: 103-118. doi:10.4172/jcsb.1000010

Copyright: () 2008 Jothi R, et al. This is an open-access article distributed under the terms of the Creative Commons Attribution License, which permits unrestricted use, distribution, and reproduction in any medium, provided the original author and source are credited.

\section{Abstract}

Whole genome sequences of the four strains of Streptococcus pneumoniae, encapsulated TIGR4, D39, G54 and nonencapsulated R6 are considered for the comparative study on genome features, whole genome pairwise alignment, gene role category, and virulence factors using relevant comparative genomics tools. The study of capsular polysaccharide synthesizing genes reveals that many cps genes are unique to TIGR4, which shows the high virulence nature of TIGR4. Further, the study on the other virulence factors such as pneumococcal surface protein A, autolysin, hyaluronate lyase, pneumolysin, neuraminidase B, and pneumococcal surface antigen $A$ of TIGR4 are much related to those of the other three strains, and hence the virulence nature due to these factors among four strains seems to be similar. But it differs from neuraminidase A, choline binding protein A and immunoglobulin A1 protease. Also in the present study, 4 and 22 hypothetical protein sequences of TIGR4 and R6 respectively are predicted as virulence factors. Among those sequences, it is found that 8 hypothetical protein sequences with 7 different functional regions of $\mathrm{R6}$ are related to other previously known virulence factors of TIGR4 and R6 of S. pneumoniae.

Keywords: Comparative genomics; Streptococcus pneumoniae; TIGR4; D39; G54; R6; Virulence factors; Hypothetical protein sequences

Abbreviations: CMR: Comprehensive Microbial Resource; CPS: Capsular Polysaccharide; PspA: Pneumococcal surface protein A; LytA: autolysin; Hyl: Hyaluronate lyase; Ply: Pneumolysin; NanA and NanB; Neuraminidases A and B; CbpA: Choline binding protein A; PsaA: Pneumococcal surface antigen A; IgA1: Immunoglobulin A1 protease

\section{Introduction}

The whole genome sequences of bacteria of closely related species or strains are providing new avenues of investigation for the further understanding of microbial diversity, pathogenesis, host-parasite interaction, evolution, etc. through a comparative analysis of their genomes. Streptococcus pneumoniae, commonly pneumococcus (Dowson, 2004; Gregory and DeSalle, 2005), a human pathogen, causes life threatening diseases like pneumoniae, bacteremia, meningi- tis, sepsis, and otitis media. Genome sequencing of four $S$. pneumoniae strains, namely, TIGR4, D39, G54 and R6 have been completed and genome sequencing of other 14 strains are ongoing. G54 genome sequence is not yet added in GenBank but it is inbuilt in Comprehensive Microbial Resource (CMR) and D39 genome sequence is available in GenBank but not in CMR. TIGR4, a clinical isolate, is encapsulated and highly virulent and many of its virulence fac- 


\section{Journal of Computer Science \& Systems Biology - Open Access}

Research Article

JCSB/Vol.1 2008

tors have been studied (Tettelin et al., 2001). D39, the encapsulated and virulent strain (Lanie et al., 2007), was used by Avery, Macleod, and McCarty (Avery et al., 1979) in their landmark study on the role of DNA as the genetic material. G54 is an encapsulated clinical strain type 19F (Dopazo et al., 2001). R6, a derivative of the serotype 2 clinical isolate D39, is nonencapsulated and avirulent. The genes encoding many virulence factors are present in R6 genome in addition to the genes of capsular biosynthesis (Hoskins et al., 2001).

Many types of comparative studies (Tettelin et al., 2001; Lanie et al., 2007; Hoskins et al., 2001; AlonsoDeVelasco et al., 1995; Brückner et al., 2004; Ferretti et al., 2004; Silva et al., 2006) have already been carried out in Streptococcus strains on various aspects. The preliminary comparative analysis (Jothi et al., 2007) of the whole genomes of both the encapsulated TIGR4 and nonencapsulated R6 strains of $S$. pneumoniae provided some insights into the high virulence nature of TIGR4. This present study summarizes specifically how the whole genomes of the four strains, namely, TIGR4, D39, G54 and R6 of S. pneumoniae differ from each other by their genome features, genome diversity, gene role category and virulence factors. Comparison of the virulence factors among these strains can provide further insight into any strain uniqueness with relevance to virulence nature and can stimulate new approaches into disease prevention and treatment.

S. pneumoniae has two surface layers outside the plasma membrane, namely, cell wall and capsule. The cell wall has triple-layered peptidoglycan that holds the capsular and cell wall polysaccharides, and also few proteins. The capsule completely covers the inner structure of $S$. pneumoniae. The cell wall polysaccharide is common to all serotypes of S. pneumoniae, but the chemical structure of the capsular polysaccharide is serotype-specific (AlonsoDeVelasco et al., 1995). After Avery's experiment (Avery et al., 1979), the capsule has long been recognized as the major virulence factor of $S$. pneumoniae. Experimental proof for this was provided by the difference in $50 \%$ lethal dose between encapsulated and nonencapsulated strains. Encapsulated strains were found (AlonsoDeVelasco et al., 1995) to be at least $10^{5}$ times more virulent than strains lacking the capsule. Certain proteins in S. pneumoniae like pneumococcal surface protein A (PspA), autolysin (LytA), hyaluronate lyase ( $\mathrm{Hyl}$ ), pneumolysin (Ply), neuraminidases $\mathrm{A}$ and B (NanA and NanB), choline binding protein A (CbpA), pneumococcal surface antigen A (PsaA) and immunoglobulin A1 (IgA1) protease are important virulence factors (AlonsoDeVelasco et al., 1995; Jedrzejas, 2001; Rigden et al., 2003) and these could be used as potential vaccine can- didates. The preliminary identification of the surface proteins and virulence factors of $S$. pneumoniae were done by computational analysis of its genome sequences (Tettelin and Hollingshead, 2004; Gregory and DeSalle, 2005; Tettelin et al., 2001; Hoskins et al., 2001) and continued in several subsequent studies (Brückner et al., 2004; Polissi et al., 1998; Wizemann et al., 2001). Strains of S. pneumoniae are now resistant to commonly prescribed antibiotics, such as, penicillin, macrolides and fluoroquinolones (Tettelin et al., 2001). Because of the multidrug resistance nature of the $S$. pneumoniae strains, we need a deeper understanding of the virulence factors, for that the comparative genomics approach may provide more insight.

At present, only $70 \%$ of the genes in any given genome can be predicted with reasonable confidence (Bork, 2000). The remaining genes are either hypothetical (do not have any known homolog) or conserved hypothetical (homologous to genes of unknown function), because it is unclear whether they encode actual proteins. The large quantity of hypothetical protein sequences in completely sequenced genomes of organisms makes their study an enormous task. Characterization of these genes or proteins of unknown function is generally recognized as an essential step towards fully understanding the biology of the pathogenic organism and for potential targets. Few studies (Galperin and Koonin, 2004; Brown, 2005; Sivashankari and Shanmughavel, 2006) have already been carried out on hypothetical sequences. In the present study, hypothetical protein sequences of the strains TIGR4 and R6 of S. pneumoniae are analyzed to find their virulence nature using VirulentPred. Among those sequences, it is also analyzed how far the hypothetical protein sequences are related to other previously known virulence factors of TIGR4 and R6 of S. pneumoniae.

\section{Materials and methods}

Various analysis of the whole genomes of the four strains, namely, TIGR4, D39, G54 and R6 of S. pneumoniae like the whole genome alignment, comparison of gene role categories, finding the location of the virulence factors in the genome and comparison of virulence regions are carried out using the appropriate bioinformatics software tools.

\section{Sequence Retrieval and Whole Genome Pairwise Alignment}

The complete genome sequences and the list of annotated gene and protein sequences of TIGR4, D39 and R6 are retrieved from the NCBI - FTP server (ftp:// ftp.ncbi.nih.gov/genomes). We used the run-mummer3 program available in the standalone MUMmer 3.20 (http:// 
mummer.sourceforge.net/) and its built-in mummerplot for obtaining the whole genome pairwise alignment of $S$. pneumoniae strains TIGR4, D39, and R6 in different combinations. MUMmer at Comprehensive Microbial Resource (CMR) is used for the whole genome pairwise alignment of the strains TIGR4, G54 and R6 in different combinations.

\section{Comparison of the Role Category of Genes and Se- quence Analysis}

The tool in CMR database (http://cmr.tigr.org/tigr-scripts/ $\mathrm{CMR} / \mathrm{CmrHomePage.cgi),} \mathrm{the} \mathrm{role} \mathrm{category} \mathrm{piechart} \mathrm{is}$ used for the genome features and functional role category comparison of the whole genomes of TIGR4, G54 and R6. Bacterial Annotation System (BASys - http:// wishart.biology.ualberta.ca/basys) - A web server for automated bacterial genome annotation is used to know the role category for three strains TIGR4, D39 and R6, whose whole genomes are already available in it. From the prediction server of the Center for Biological Sequence Analysis (CBS - http://www.cbs.dtu.dk/services), the Genome Atlas is used for the analysis of repeats of $S$. pneumoniae. The sequences of various virulence factors, which are taken for our study, have been verified by using the virulence factors database (http://www.mgc.ac.cn/VFs). BioEdit (http:// www.mbio.ncsu.edu/ BioEdit/bioedit.html) is used to compute sequence composition of the genomes and genes. Further, LALIGN (http://www.ch.embnet.org/software/ LALIGN_form.html) is used for the pairwise global alignment of the gene sequences of the strains of S. pneumoniae.

\section{Functional Annotation of Hypothetical Sequences}

VirulentPred (http://bioinfo.icgeb.res.in/virulent) is a SVM (Support Vector Machine) based method to predict bacterial virulent protein sequences, which can be used to screen virulent proteins in proteomes. In the present study the above tool is used to analyse the hypothetical sequences of the strains TIGR4 and R6 of $S$. pneumoniae. From the proteome of TIRG4 and R6 of $S$. pneumoniae, all unannotated hypothetical protein sequences are retrieved using PERL script and those sequences are used as data set for virulence factor prediction.

\section{Results and Discussion}

Comparative genomics and in silico studies have begun to reveal insights into gene and protein functions of many organisms. Here, we compare the genomes of the strains TIGR4, D39, G54 and R6 of S. pneumoniae using the appropriate tools for whole genome comparison and the results are discussed below.

\section{Comparison of the Genome Features of Four Strains of $S$. Pneumoniae}

Table 1 summarizes the general information about the genomes including statistics of genes of these four strains, obtained and compiled from CMR and NCBI web servers. The genome sizes of these four strains range between 2 $\mathrm{Mb}$ and $2.16 \mathrm{Mb}$ (c.f. S1.No.2 of Table1). Among these four strains, D39 is the smallest and TIGR4 is the largest based on genome size. The nucleotide base (A, T, G, C, AT and GC) compositions of four strains show that the strains have low GC ( 40\%) genomes. The number of genes encoding for proteins of these four strains ranges between 1914 and 2234 (c.f. Sl.No.3 of Table1). Of the total base pairs of four genomes, approximately $85-87 \%$ of base pairs (bps) are involved in coding and the remaining are non-coding or junk DNA. The number of genes involved in RNA synthesis (structural RNA, tRNA, and rRNA) is more or less similar in all strains. Finally, by comparing the global and local repeats of TIGR4 and R6 using CBS web server, it is evident that both the repeats are high in TIGR4 than in R6 (c.f. S1.No.4 of Table1) and this may be related to the duplicated regions of the chromosome (Gregory and DeSalle, 2005).

\section{Comparison of Whole Genome Pairwise Alignments}

The whole genome pairwise alignments of the strains TIGR4, D39 and R6 of S. pneumoniae (whose sequence data are available at NCBI) are obtained using the standalone version of MUMmer and the results are plotted using its built-in mummerplot. The whole genome pairwise alignments of the strains TIGR4, G54 and R6 are obtained using CMR, where these sequences are available, and the five possible alignments are shown in Figure 1(a) - (e). Generally, the genomes of prokaryotes are very dynamic, with insertions, deletions, inversions, and translocations being commonly observed among related species or even between different strains of the same species (Gregory and DeSalle, 2005; Hughes, 2000). The net result is that the particular complement of genes and their order along the chromosome are not typically conserved over evolutionary time. In some cases, genes that are grouped into operons in one species may be dispersed throughout the genome in others. We find similar results, while we analyzed the genomes of four strains of $S$. pneumoniae. In particular, we find that there exists a stability of the gene order in the genome pairs TIGR4 vs. D39 and TIGR4 vs. R6 and they are shown by fact that most of the points lie along the diagonal in Figures $1 \mathrm{a}$ and $1 \mathrm{~b}$. The results (Figures $1 \mathrm{a}$ and $1 \mathrm{~b}$ ) indicate that the stability of gene order of D39 vs. R6 must also be relatively high and it is shown in Figure 1c. This also confirms the 


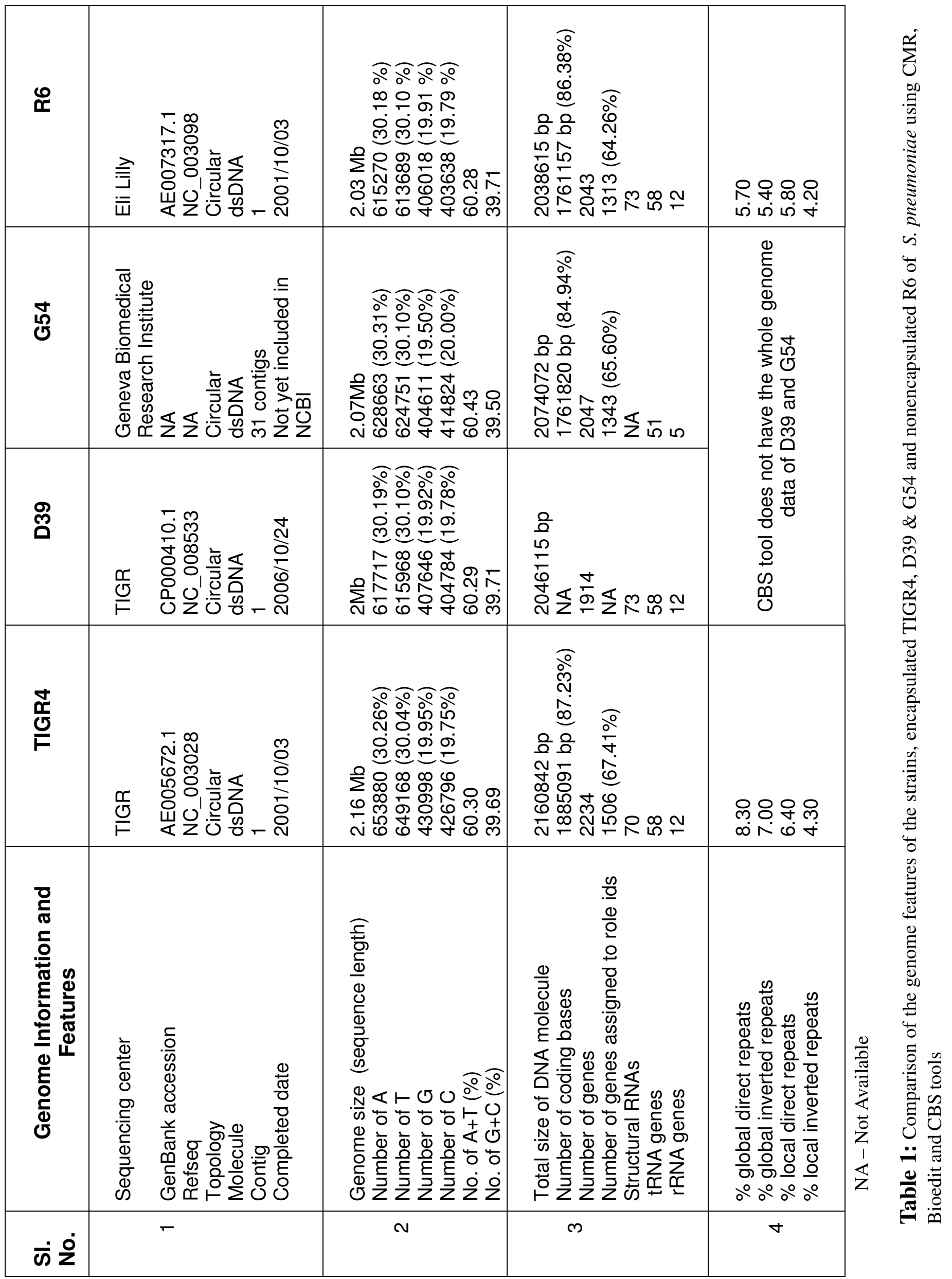



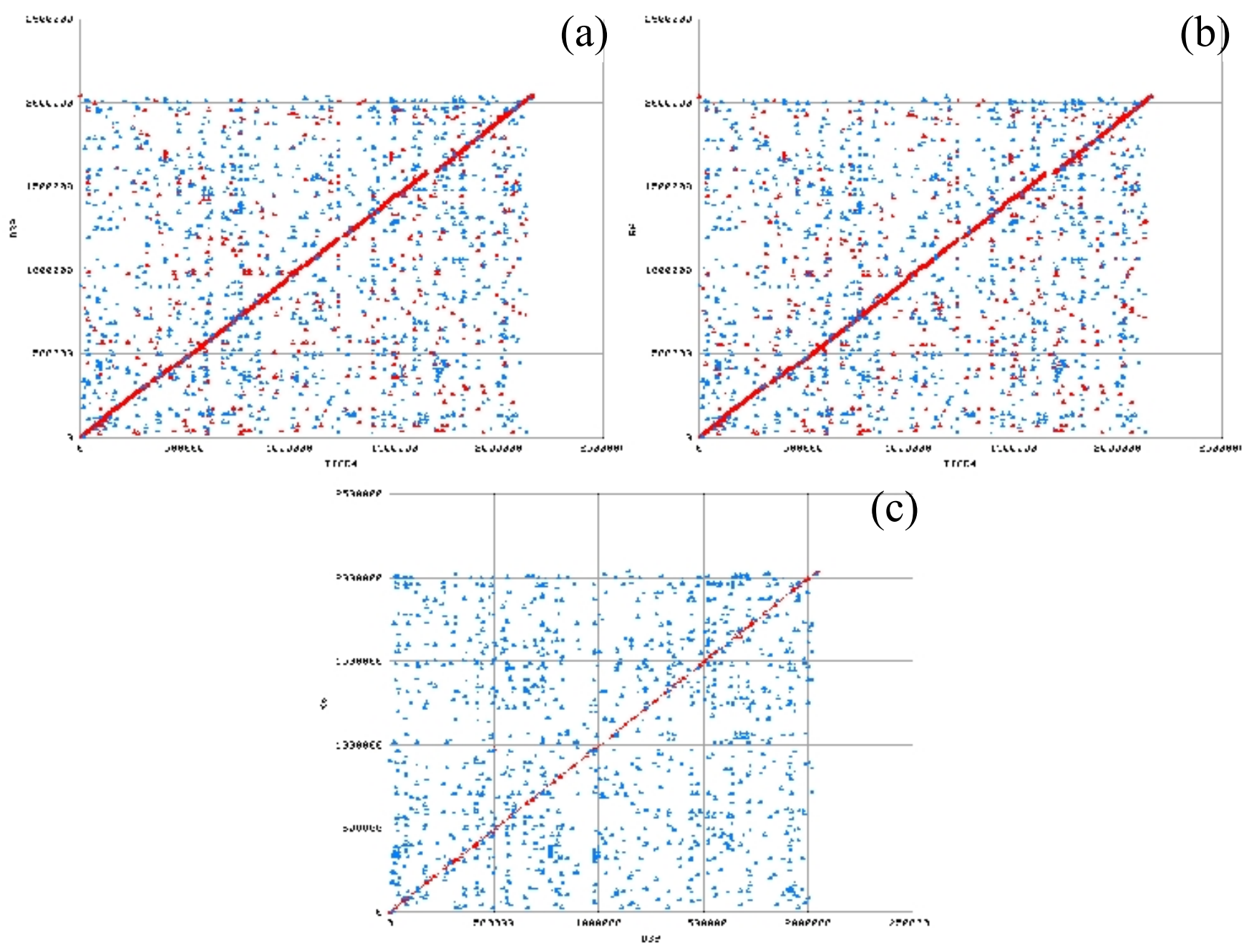

\section{(c)}
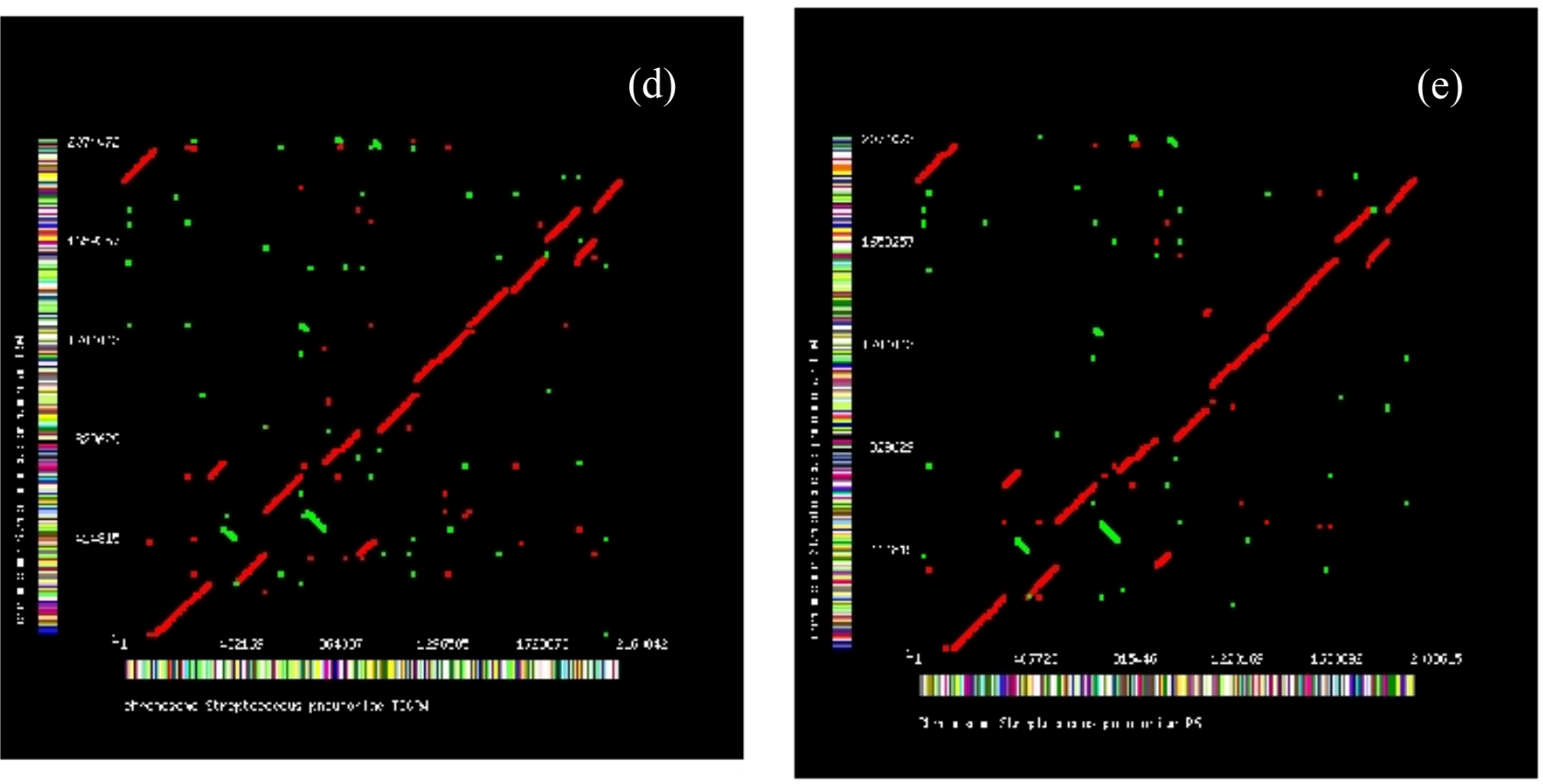

Figure 1: Whole genome alignment of a) TIGR4 vs. D39; b) TIGR4 vs. R6; c) D39 vs. R6 using stand-alone MUMmer; Whole genome alignment of d) TIGR4 vs. G54 and e) R6 vs. G54 using built-in MUMmer of CMR, which show plasticity and stability in gene order between two strains. 
fact that R6 is the derivative of D39. The whole genome pairwise alignments of TIGR4 vs. G54 and that of R6 vs. G54 do not show such a high degree of the stability of gene order compared to the above results (for D39 strain) and are shown in Figures 1d and 1e, respectively.

Many of the gene and protein sequences among these strains are approximately the same and this is not surprising as all the strains occupy the same niche in the human respiratory system. The small differences might have arisen after the divergence of these strains from other evolutionary lineages for adaptations in their host. This increases greatly in pathogens and appears to be associated with the ability to infect eukaryotes, perhaps reflecting a mechanism for evading host immune defenses and the unique genes may be located in a plasticity zone.

Since G54 genome sequence is not available at NCBI web server and D39 genome is not available at CMR server, we could not get the whole genome alignment for D39 vs. G54. However, we are able to predict the whole genome pairwise alignment of D39 vs. G54, based on the earlier result. As the Figures 1d and 1e are similar, it indicates that the alignment of D39 vs. G54 must also possess similar structure. This prediction may be confirmed if the whole genome sequence of G54 is made available in NCBI or genome sequence of D39 is included in CMR.

\section{Comparison of Capsular Polysaccharide Synthesizing Genes}

We have compared the capsular polysaccharide (cps) synthesizing genes of the strains TIGR4, D39, G54 and R6 of $S$. pneumoniae and the results are shown in Table 2. There are 15 different cps genes in TIGR4, 7 in D39 and 9 in G54 and only one in R6. Their gene IDs, G+C percentage, protein length, gene length and gene coordinates are shown in Table 2. On comparison, it is estimated that $5 \mathrm{cps}$ genes of TIGR4 (gil15900275-cps4A, gil15900276-cps4B, gil15900278-cps4D, gil15900046-cps-ptv \& gil15901666-cpsptv) are related to that of D39 (gil116516963-cps2A, gil116516159-cpsB, gil116517023-cps2D, gil116517199-cps and gil116516120-cps-ptv). All the cps genes of D39 are present in TIGR4 except gil116516773-cps2E and gil116516341-cps-ptv.

Between TIGR4 and G54, $6 \mathrm{cps}$ genes are related (gil15900275-cps4A, gil15900276-cps4B, gil15900277cps4C, gil15900278-cps4D, gil15900046-cps-ptv \& gil15901666-cps-ptv of TIGR4 with NT05SP0190-cps4A, NT05SP0191-cps4B, NT05SP0192-cps4C, NT05SP0193cps4D, NT05SP2185-cps9E \& NT05SP1650-cps7G of
G54). Likewise, between D39 and G54, 5 cps genes are related (gil116516963-cps2A, gil116516159-cpsB, gil116517023-cps2D, gil116517199-cps and gil116516120cps-ptv of D39 with NT05SP0190-cps4A, NT05SP0191cps4B, NT05SP0192-cps4C, NT05SP2185-cps9E \& NT05SP1650-cps7G of G54), but gil116516773-cps2E and gil116516341-cps-ptv of D39 are not present in G54. Similarly, it is interesting to note that the only cps gene of R6 (gil15902136-capD), has $99.8 \%$ identity with the gene gil15900046-cps-ptv of TIGR4, $100 \%$ identity with the gene gil116517199-cps of D39 and $99.5 \%$ identity with the gene NT05SP2185 of G54. All the above results are in support of the Avery's statement (Avery et al., 1979) that the capsule is responsible for pathogenecity.

From similar analysis, we have also noted that the genes, gil15900279-cps4E, gil15900280-cps4F, gil15900281-cps4G, gil15900282-cps4H, gil15900286-cps4I, gil15900287-cps4J, gil15900288-cps4K, gil15900289-cps4L and gil15900788cps-ptv are unique to TIGR4. Similarly, the genes gil116516773-cps2E and gil116516341-cps-ptv are unique to D39 strain. In the same way, the genes NT05SP0198, NT05SP0202 and NT05SP1909 are unique to the strain G54. But in R6, the only cps gene gil15902136-capD is common to all other strains (Table 2). As the TIGR4 strain has more number of cps genes than other strains it indicates the high virulence nature of TIGR4. Further, the results also explain that the virulence nature is lesser in D39 and G54 strains, and very less in R6 compared to TIGR4.

Though all the cps genes of TIGR4 are not present in D39, G54 and R6 strains, they are also pathogenic. Therefore, to know the other virulence factors in addition to cps genes, we consider the other genes of the strains from the gene role category aspect.

\section{Comparison of the Role Category of Genes}

Role category of genes of the different strains are compared by using the two different tools, namely, i. CMR role category pie chart for TIGR4, G54 and R6 (Table 3) and ii. Bacterial Annotation System (BASys) for the strains TIGR4, D39 and R6, based on the availability of genome sequences. The genes responsible for biosynthesis of various proteins (Sl. Nos. 1-9 of Table 3) of TIGR4 are nearly same as in G54 and R6, which suggests the basic complement of proteins required for certain cellular processes. But the genes responsible for the biosynthesis of some other proteins (Sl.Nos.10-23 of Table 3) of TIGR4 are notably different from that of G54 and R6. This suggests that, these proteins are important for strain uniqueness and they may be involved in variations in pathogenesis among the strains 
Journal of Computer Science \& Systems Biology - Open Access

Research Article

JCSB/Vol.1 2008

\begin{tabular}{|c|c|c|c|c|c|c|}
\hline $\begin{array}{l}\text { Strain } \\
\text { name }\end{array}$ & Gene ID and Name & $\begin{array}{l}\mathrm{G}+\mathrm{C} \\
(\%)\end{array}$ & $\begin{array}{l}\text { Protein } \\
\text { length } \\
\text { (aa) }\end{array}$ & $\begin{array}{l}\text { Gene } \\
\text { length } \\
\text { (bp) }\end{array}$ & $\begin{array}{c}\text { Gene } \\
\text { coordinates }\end{array}$ & $\begin{array}{c}\text { Comparison with cps } \\
\text { of other strains } \\
\text { in \%ldentity }\end{array}$ \\
\hline TIGR4 & $\begin{array}{l}\text { gi|15900275-cps4A } \\
\text { gi|15900276-cps4B } \\
\text { gi|15900277-cps4C } \\
\text { gi|15900278- cps4D } \\
\text { gi|15900279-cps4E } \\
\text { gi|15900280-cps4F } \\
\text { gi|15900281-cps4G } \\
\text { gi|15900282-cps4H } \\
\text { gi|15900286-cps4I } \\
\text { gi|15900287-cps4J } \\
\text { gi|15900288-cps4K } \\
\text { gi|15900289-cps4L } \\
\text { gi|15900046-cps-ptv* } \\
\text { gi|15900788-cps-ptv } \\
\text { gi|15901666-cps-ptv }\end{array}$ & $\begin{array}{l}38.32 \\
41.98 \\
\\
40.29 \\
34.21 \\
\\
33.49 \\
33.17 \\
27.84 \\
31.36 \\
36.70 \\
38.46 \\
36.19 \\
35.02 \\
42.21 \\
\\
\\
28.79 \\
43.93\end{array}$ & $\begin{array}{l}481 \\
243 \\
230 \\
227 \\
211 \\
409 \\
358 \\
372 \\
365 \\
351 \\
409 \\
394 \\
616\end{array}$ & $\begin{array}{r}1446 \\
732 \\
693 \\
684 \\
\\
636 \\
1230 \\
1077 \\
1119 \\
1098 \\
1056 \\
1230 \\
1185 \\
1851 \\
\\
1368 \\
1227\end{array}$ & $\begin{array}{l}320077-321522 \\
321524-322255 \\
322264-322956 \\
322966-323649 \\
\\
323990-324625 \\
324634-325863 \\
325868-326944 \\
326937-328055 \\
331774-332871 \\
332875-333930 \\
334030-335259 \\
335260-336444 \\
104668-106518 \\
\\
859370-860737 \\
1746322-1747548\end{array}$ & $\begin{array}{c}96.0 \text { - D39-gi|116516963-cps2A } \\
94.0 \text { - G54-NT05SP0190-cps4A } \\
97.9 \text { - D39-gi|116516159-cpsB } \\
86.4 \text { - G54-NT05SP0191-cps4B } \\
\text { 85.7 - G54-NT05SP0192-cps4C } \\
\text { 79.6 - D39-gi|116517023-cps2D } \\
93.8 \text { - G54-NT05SP0193-cps4D } \\
-- \\
-- \\
-- \\
-- \\
-- \\
-- \\
-- \\
99.8 \text { - D39-gi|116517199-cps } \\
99.7 \text { - G54-NT05SP2185-cps9E } \\
99.8 \text { - R6-gi|15902136-capD } \\
-- \\
99.0 \text { - D39-gi|116516120-cps-ptv } \\
96.6 \text { - G54-NT05SP1650-cps7G }\end{array}$ \\
\hline D39 & $\begin{array}{l}\text { gi|116516963-cps2A } \\
\text { gi|116516159-cpsB } \\
\text { gi|116517023-cps2D } \\
\text { gi|116516773-cps2E } \\
\text { gi|116517199-cps } \\
\text { gi|116516341-cps-ptv } \\
\text { gi|116516120-cps-ptv }\end{array}$ & $\begin{array}{l}38.45 \\
41.53 \\
39.06 \\
37.79 \\
42.19 \\
\\
30.28 \\
44.09\end{array}$ & $\begin{array}{l}481 \\
243 \\
226 \\
455 \\
616 \\
\\
119 \\
408\end{array}$ & $\begin{array}{r}1446 \\
732 \\
681 \\
1368 \\
1851 \\
\\
360 \\
1227\end{array}$ & $\begin{array}{r}313744-315189 \\
315191-315922 \\
316633-317313 \\
317328-318695 \\
99217-101067 \\
\\
815811-816170 \\
1633887-1635113\end{array}$ & $\begin{array}{c}96.3 \text { - G54-NT05SP0190-cps4A } \\
85.2 \text { - G54-NT05SP0191-cps4B } \\
79.3 \text { - G54-NT05SP0192-cps4C } \\
-- \\
99.5 \text { - G54-NT05SP2185-cps9E } \\
100 \text { - R6-gi|15902136-capD } \\
-- \\
97.1 \text { - G54- NT05SP1650-cps7G }\end{array}$ \\
\hline G54 & $\begin{array}{l}\text { NT05SP0190-cps4A } \\
\text { NT05SP0191-cps4B } \\
\text { NT05SP0192-cps4C } \\
\text { NT05SP0193-cps4D } \\
\text { NT05SP0198-cps19AI } \\
\text { NT05SP0202-cps23FP } \\
\text { NT05SP1650-cps7G } \\
\text { NT05SP1909-cps3E } \\
\text { NT05SP2185-cps9E }\end{array}$ & $\begin{array}{l}38.28 \\
37.56 \\
38.09 \\
34.64 \\
29.82 \\
41.70 \\
43.77 \\
43.63 \\
42.46\end{array}$ & $\begin{array}{l}484 \\
243 \\
230 \\
227 \\
445 \\
198 \\
417 \\
436 \\
616\end{array}$ & $\begin{array}{r}1455 \\
732 \\
693 \\
684 \\
1338 \\
597 \\
1254 \\
1311 \\
1851\end{array}$ & $\begin{aligned} & 165975-167429 \\
& 167431-168162 \\
& 168171-168863 \\
& 168873-169556 \\
& 173388-174725 \\
& 178230-178826 \\
& 1493392-1492139 \\
& 1726013-1727323 \\
& 1999333-2001183\end{aligned}$ & $\begin{array}{c}-- \\
-- \\
-- \\
-- \\
-- \\
-- \\
-- \\
-- \\
99.5-\text { R6-gi|15902136-capD }\end{array}$ \\
\hline R6 & gi|15902136-capD & 42.26 & 616 & 1851 & $99217-101067$ & -- \\
\hline
\end{tabular}

Table 2: Comparison of capsular polysaccharide (cps) synthesizing genes of four strains of S. pneumoniae. Each cps is compared with all cps sequences of other three strains using LALIGN; all the cps sequences considered fall under the Role Category 11 (Cell Envelope) of CMR.

of $S$. pneumoniae. The percentage values given for a particular role category in Table 3 is specific to the gene involved in that category only and does not represent the overall gene percentage. For example, autolysin (SP1937) of
TIGR4 is categorized into two role categories such as cell envelope and cellular processes (Sl.Nos.11 and 12 of Table 3 ) and the percentage given is specific to the respective categories. 


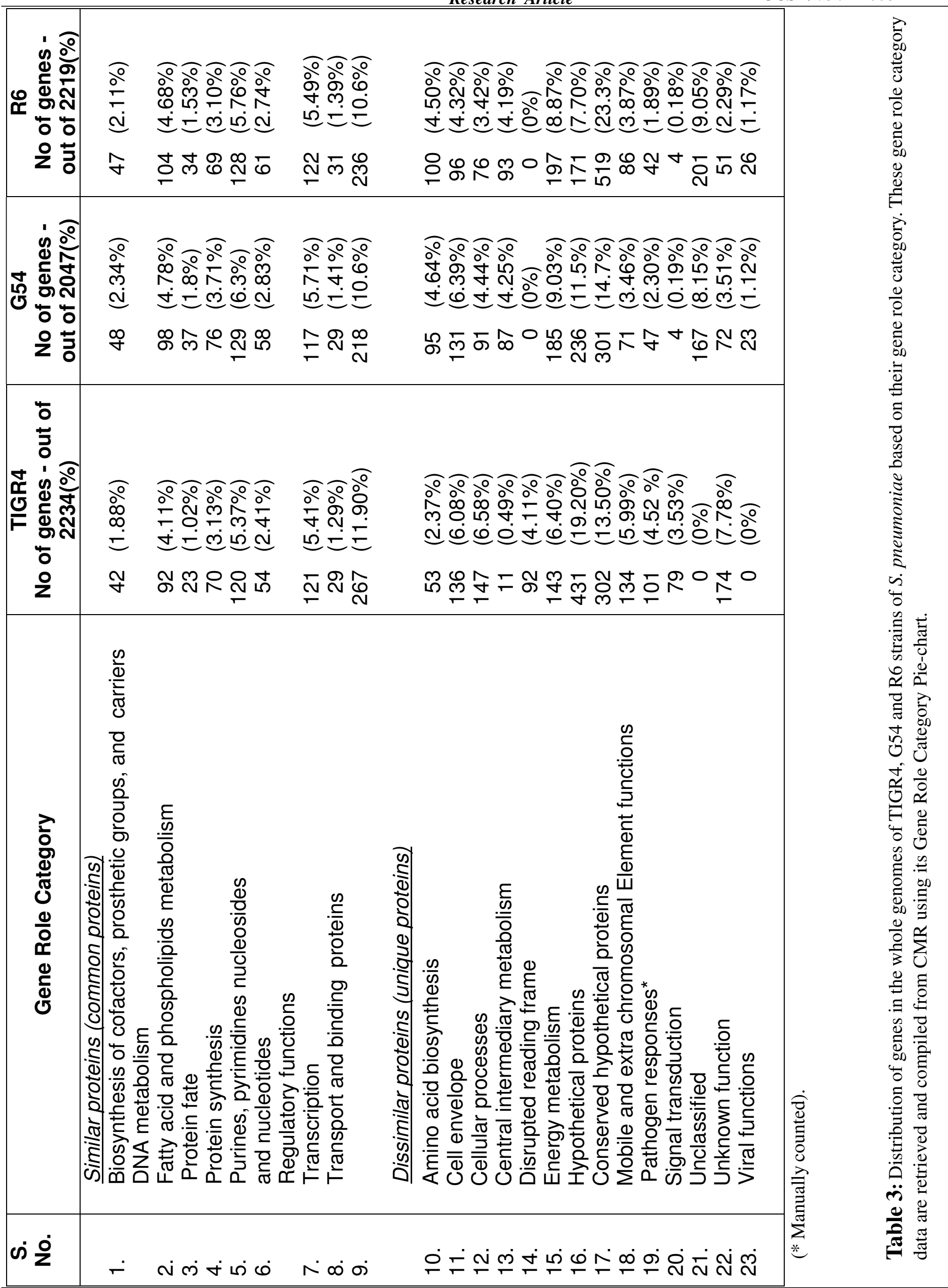




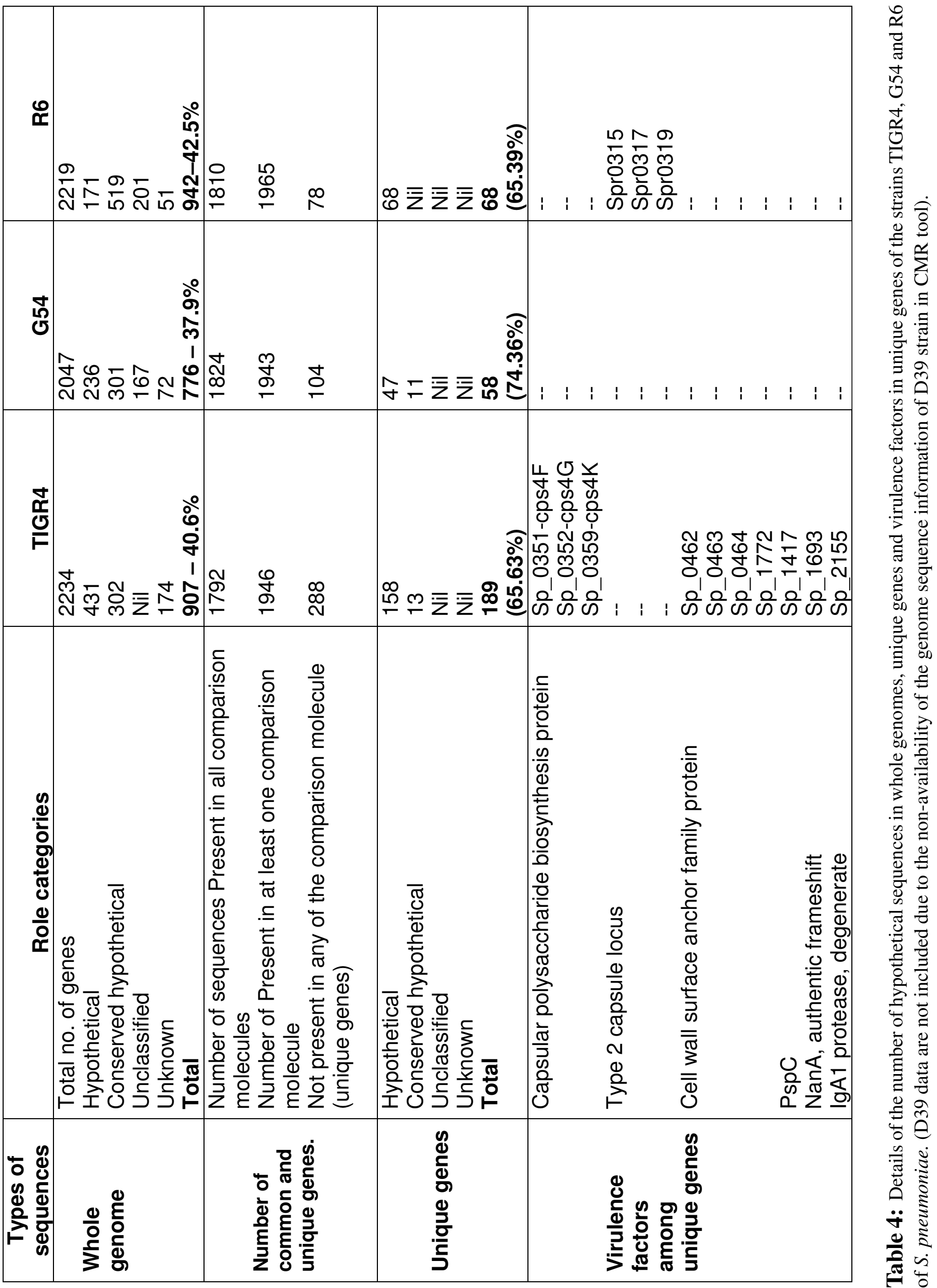


The number of genes which are responsible for pathogenesis in the strains TIGR4, G54 and R6 are manually counted from CMR gene role category (sub role categories pathogenesis, toxin production and resistance) and found to be $101(4.52 \%), 47(2.30 \%)$ and $42(1.89 \%)$ respectively (Sl.No.19 of Table 3). TIGR4 has many pathogenic factors and it is highly virulent and G54 and R6 strains have approximately $50 \%$ of the pathogenic factors of TIGR 4 . Mobile and extra chromosomal elements comprise a significant fraction of the genome as with the 134 genes (5.99 $\%)$ in TIGR4, 71 (3.46\%) in G54 and 86 genes (3.87\%) in R6 (Sl.No.18 of Table 3). Generally transposons encode genes for antibiotic resistance (Gregory and DeSalle, 2005); therefore from our results, it is evident that the antibiotic resistance may be relatively higher in TIGR4 than the strains G54 and R6.

From the results of the comparative study on TIGR4, D39 and R6, using BASys server, we find that most of the values are more or less similar. But, there is a higher percentage for unknown functions in the strains TIGR4, D39 and G54, which indicates that the reason for the differences may also be hidden in the unknown genes or proteins (data not shown).

From Table 3, the number of hypothetical, conserved hypothetical, unclassified and unknown genes of whole genomes of the strains TIGR4, G54 and R6 are noted and is shown in Table 4. Nearly $37-42 \%$ of genes are of unknown type and it shows that these sequences have to be annotated and assigned functions of which some of them may be responsible for the virulence nature. Using the multigenome homology comparison tool, which is available at CMR, the numbers of unique genes in TIGR4, G54 and R6 are found to be 288, 104 and 78, respectively (Table 4).

The unique genes of the strains TIGR4, G54 and R6 themselves have many hypothetical, conserved hypothetical, unknown and unclassified sequences and their percentage ranges from 65 to 74 , thus the other possible differences among the strains may be known by studying the above said gene sequences. As far as the virulence factors are concerned, in the unique genes of the strain TIGR4, 3 capsular polysaccharide biosynthesis proteins (Sp_0351 (cps4F), Sp_0352 (cps4G) and Sp_0359 (cps4K)), 4 cell wall surface anchor family proteins (Sp_0462, Sp_0463, Sp_0464 and Sp_1772), a PspC protein (Sp_1417), a NanA protein (SP_1693) and a IgA1 protease (SP_2155) are there. In the case of R6, it has three proteins of type 2 capsule locus (Spr0315, Spr0317 and Spr0319) in its unique genes. But the strain G54 does not have such virulence factors in its unique genes (Table 4). The above result shows the high virulence nature of TIGR4 and it also suggests that those virulence factors are specific to TIGR4 and R6. The above differences might have arisen because of the species-specific adaptation to their host particularly in the sake of defense mechanism.

\section{Comparison of Virulence Factors Other than Capsu- lar Polysaccharide Synthesizing Genes}

In S. pneumoniae, the surface and cytoplasmic proteins such as pneumococcal surface protein A (PspA), autolysin (LytA), hyaluronate lyase (Hyl), pneumolysin (Ply), two neuraminidases (NanA and NanB), choline binding protein A ( CbpA), pneumococcal surface antigen A (PsaA) and immunoglobulin A1 (IgA1) protease are already stated as the virulence factors (Jedrzejas, 2001; Rigden et al., 2003). The comparative results of the above mentioned sequences obtained from CMR, are given in Table 5. It provides more insight into the virulence factors of the strains TIGR4, D39, G54 and R6 of S. pneumoniae.

The virulence factors of TIGR4 are taken as reference and are compared with all other related sequences of the strains such as D39, G54 and R6, likewise the virulence factors of D39 are taken as reference and are compared with all the related sequences of the strains G54 and R6. Similarly the virulence factors of G54 are taken as reference and are compared with all the related sequences of the remaining strain R6 using the pairwise sequence alignment tool LALIGN, with default parameters (Alignment: Global; Scoring matrix: BLOSUM50, Gap opening penalty: -14 and extension penalty: -4 ), and all the results are comparatively shown in Table 5 .

PspA is located in the cell wall of pneumococci and present in all S. pneumoniae strains (Jedrzejas, 2001). PspA of TIGR4 has 53-63\% identities with D39, G54 and R6 (Table 5). When we compare PspA in D39 vs. G54 and G54 vs. R6, the identities between those strains are nearly $63 \%$. The above results indicate that nearly $50-60 \%$ virulence nature of PspA of TIGR4 exist in other strains D39, G54 and R6. But it is interesting to note that there is $100 \%$ identity between the PspA sequences of D39 and R6, thus the virulence nature of PspA is exactly the same.

Regarding LytA, Hyl, Ply, NanB and PsaA, all the four strains of S. pneumoniae have above $90 \%$ identities, thus the effect of the above mentioned five virulence factors is also similar and it also reflects on $\mathrm{G}+\mathrm{C}$ percentage, protein length and gene length, but the location in their genomes varies and the similarities and differences can be noticed from the Table 5. 
Journal of Computer Science \& Systems Biology - Open Access

Research Article

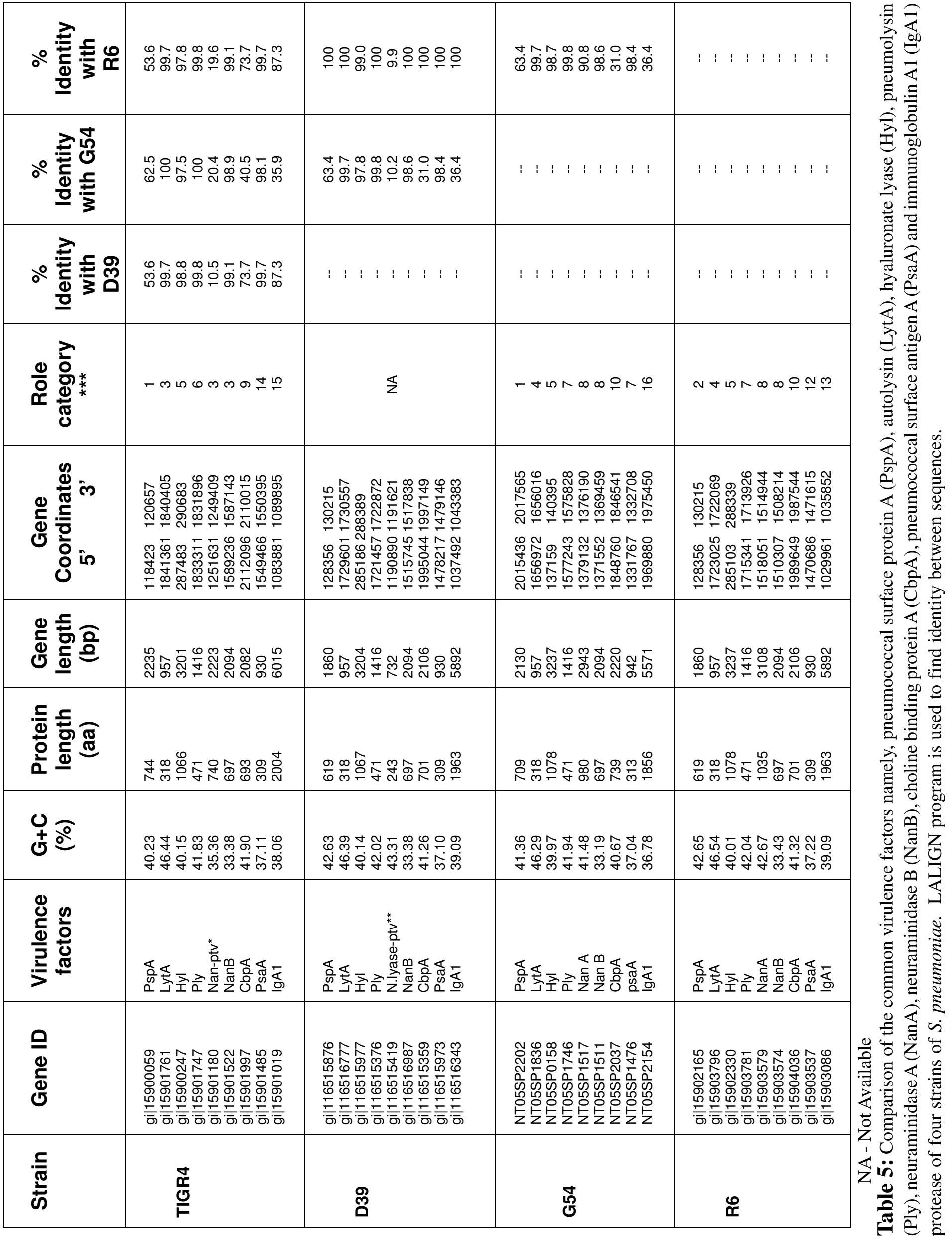


* Nan-ptv: Neuraminidase, putative

** N.lyase-ptv: N-acetylneuraminate lyase, putative

\section{*** Role category functions}

1. Cell envelope; cellular process - pathogenesis

2. Mobile and extra chromosomal element function: transposon function

3. Cell envelope biosynthesis and degradation of surface polysaccharides and Lipopolysaccharides; Cellular processes: pathogenesis

4. Cell envelope: biosynthesis and degradation of murine sacculus and peptidoglycan

5. Cellular processes: pathogenesis

6. Cellular processes: toxin production and resistance; Cellular processes: pathogenesis

7. Unclassified: role category not yet assigned

8. Viral function: general

9. Cell envelope; cellular process - pathogenesis cellular process: cell adhesion

10. Cellular processes toxin production and resistance; Fatty acid and phospholipid metabolism: degradation

11. Cell envelope biosynthesis and degradation of surface polysaccharides and Lipopolysaccharides

12. Unclassified - role category not yet assigned

13. protein fate: Degradation of proteins, peptides and glycopeptides

14. Transport and binding proteins: Cations and iron carrying compounds; Cellular processes: pathogenesis; cellular processes:cell adhesion

15. protein fate: Degradation of proteins, peptides and glycopeptides; Cellular processes: pathogenesis

16. protein fate: Degradation of proteins, peptides and glycopeptides

All strains have different neuraminidase sequences except G54 and R6 ( $~ 90 \%$ identity). In the case of CbpA and IgA1 of the strain TIGR4, high percent identities ( 73 and $87 \%$ ) exist with D39 and R6 respectively, exactly identical (100\%) between D39 and R6. But very less identities ( 40 and 35\%) exist with G54 combinations. It seems that the virulence nature based on cbpA and IgaA are similar among the strains TIGR4, D39 and R6 and differs in G54.

From Table 5, it is interesting to note that all the virulence factors of D39 are very similar to R6 (above 99\% identities except NanA), and it confirms the fact that the avirulent strain R6 is the derivative of the strain D39 (Lanie et al., 2007). Based on the role category, all TIGR4 virulence factors come under pathogenesis related functions and it also says that TIGR4 has high virulence nature.

\section{Functional Annotation of Hypothetical Sequences Relevant to the Virulence Factors}

Prediction of virulence factors from the hypothetical sequences of $S$. pneumoniae has implications on the identification and characterization of the virulence mechanism. The present study predicted using VirulentPred (Garg and Gupta, 2008) that 4 hypothetical sequences of TIGR4 and 22 of R6, respectively, are virulence factors. All these sequences are listed in Table 6. The prediction is based on protein features, such as, amino acid composition, di-peptide composition, similarity search, higher order di-peptide composition, PSSM and cascaded SVM module of the tool VirulentPred. However, similar predictions are not possible at present with D39 and G54 as the sequence information of the latter is not fully available.

Among the 4 predicted virulence factors of TIGR4, only one sequence (gil15901572) is predicted in R6 as a hypothetical protein (gil15903627) and the functional region is predicted as Plasmid_Txe (PF06769). This family contains many hypothetical proteins and there is no homolog with other mentioned virulence factors. But in R6, it is interesting to note that among the 22 predicted virulence factors of hypothetical protein sequences, 8 different sequences (gil15902372, gil15903388, gil15903446, gil15902652, gil15902781, gil15903694, gil15903627 and gil15903771) with 7 different functional regions which are related to the already mentioned virulence factors of the strains R6 and TIGR4. Those virulence factors are hyaluronidase, Immunoglobulin A1 protease, capsular polysaccharide synthesis, pneumolysin, neuraminidase and choline binding protein. The above mentioned related sequences of TIGR4 and R6 except gil15903771 are compared in Table 7.

The hypothetical protein sequence, gil15903771 of R6 has 71 amino acids and its functional region is predicted as pu- 


\begin{tabular}{|l|l|l|}
\hline $\begin{array}{c}\text { S. } \\
\text { No. }\end{array}$ & \multicolumn{1}{|c|}{ Protein ID } & \multicolumn{1}{|c|}{$\begin{array}{c}\text { Protein } \\
\text { Length }\end{array}$} \\
\hline \multicolumn{3}{|c|}{ TIGR4 } \\
\hline 1 & gi|15900762 & 177 \\
\hline 2 & gi|15900877 & 1039 \\
\hline 3 & gi|15901572 & 84 \\
\hline 4 & gi|15902036 & 255 \\
\hline \multicolumn{3}{|c|}{ R6 } \\
\hline 1 & gi|15902135 & 385 \\
\hline 2 & gi|15902152 & 450 \\
\hline 3 & gi|15902269 & 65 \\
\hline 4 & gi|15902355 & 57 \\
\hline 5 & gi|15902369 & 149 \\
\hline 6 & gi|15902372 & 1767 \\
\hline 7 & gi|15902511 & 111 \\
\hline 8 & gi|15902652 & 337 \\
\hline 9 & gi|15902781 & 170 \\
\hline 10 & gi|15902826 & 177 \\
\hline 11 & gi|15902850 & 122 \\
\hline 12 & gi|15903009 & 368 \\
\hline 13 & gi|15903331 & 330 \\
\hline 14 & gi|15903388 & 202 \\
\hline 15 & gi|15903446 & 2551 \\
\hline 16 & gi|15903447 & 502 \\
\hline 17 & gi|15903627 & 84 \\
\hline 18 & gi|15903694 & 719 \\
\hline 19 & gi|15903697 & 243 \\
\hline 20 & gi|15903771 & 71 \\
\hline 21 & gi|15903873 & 64 \\
\hline 22 & gi|15903916 & 380 \\
\hline
\end{tabular}

Table 6: List of predicted 4 and 22 hypothetical protein sequences as virulence factors from Tigr4 and R6 respectively.

tative cell wall binding repeat (42-60) using Interproscan (ID - PF01473). It is also found that the same functional region is repeatedly present in the known virulence factors such as pneumococcal surface protein A, autolysin and choline binding proteins of the strains TIGR4 and R6. Since many domain regions have been identified in the above mentioned known virulence factors of TIGR4 and R6, the regions are not explicitly given. But one can easily obtain those regions using the tool Interproscan.

\section{Conclusion}

We have compared the virulence nature of the strains, encapsulated TIGR4, D39, G54 and nonencapsulated R6 of Streptococcus pneumoniae using comparative genomics tools. From the whole genome pairwise alignment, we found that the stability of the gene order in the genomes of TIGR4 vs. D39, TIGR4 vs. R6 and D39 vs. R6 are relatively higher than the genomes of TIGR4 vs. G54 and R6 vs. G54. We are able to predict the possible structure of whole genome pairwise alignment of D39 vs. G54 from the alignments of TIGR4 vs. G54 and R6 vs. G54.

From the comparison on the capsular polysaccharide (cps) synthesizing genes, we found that, TIGR4 strain has more number of cps genes than other strains, which may indicate the high virulence nature of TIGR4. Many cps genes are unique to TIGR4, only few are in D39 \& G54 and none in R6, which shows the high virulence nature of TIGR4. Further, the study on other virulence factors such as, pneumo- 


\begin{tabular}{|c|c|c|c|c|c|c|c|}
\hline 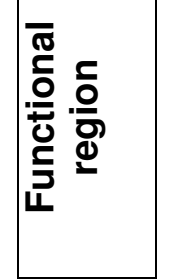 & 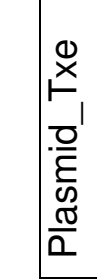 & 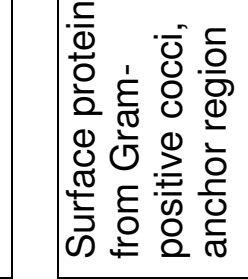 & 竞: & 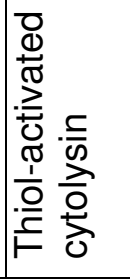 & 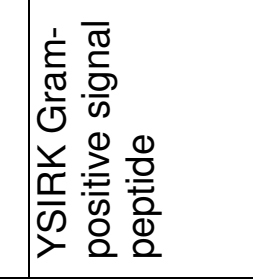 & டி & 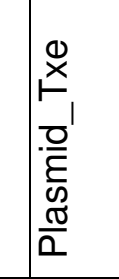 \\
\hline 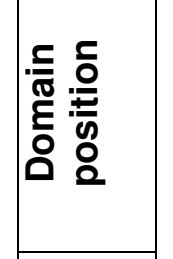 & 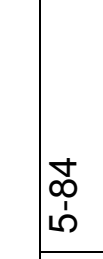 & 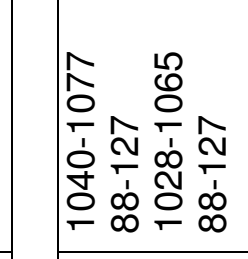 & 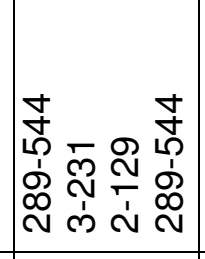 & 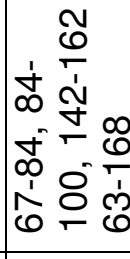 & 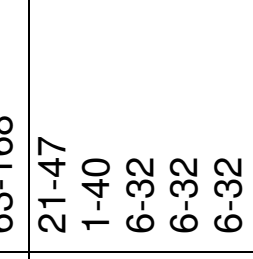 & 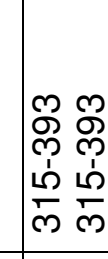 & 歺 \\
\hline 点 & \$ & $\begin{array}{l}0 \\
0 \\
0\end{array}$ & 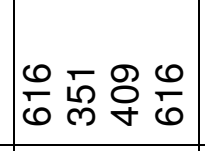 & \begin{tabular}{ll}
$\sqrt{2}$ & \multicolumn{2}{|c}{}
\end{tabular} & 挮 & & :̦t \\
\hline 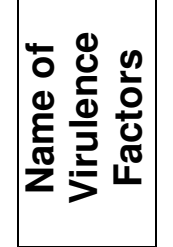 & 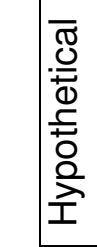 & 촛흐조점 & 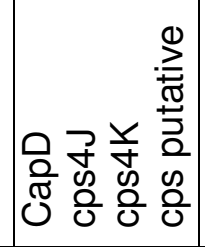 & $\geq \quad \geq$ & 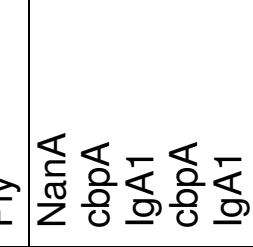 & & 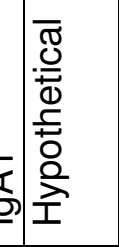 \\
\hline 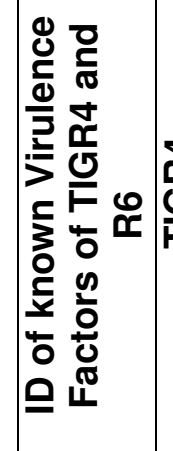 & 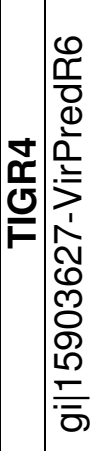 & 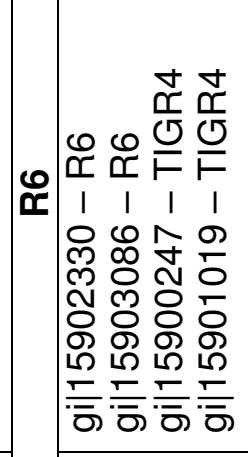 & 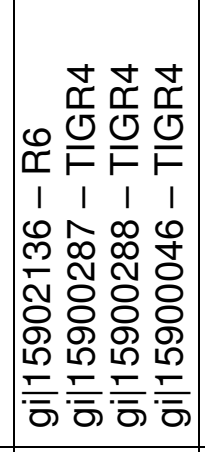 & 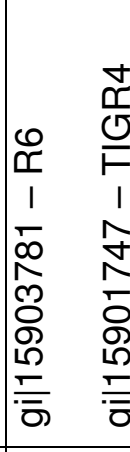 & 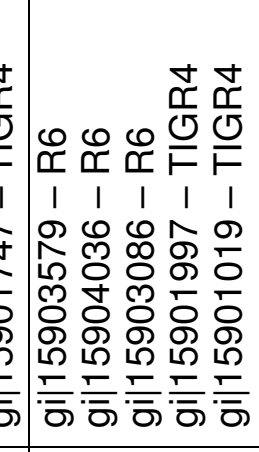 & 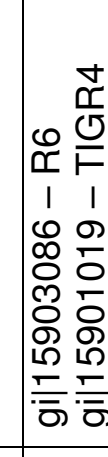 & 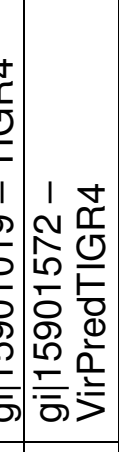 \\
\hline 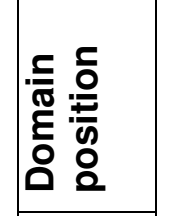 & 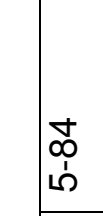 & 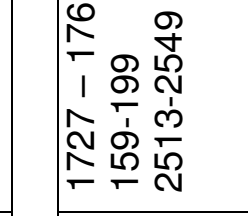 & 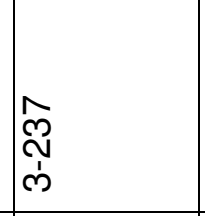 & 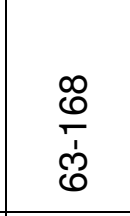 & 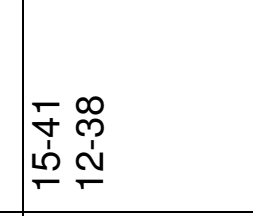 & 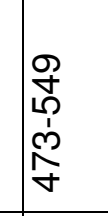 & 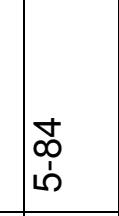 \\
\hline 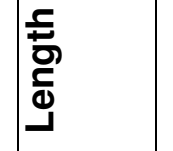 & 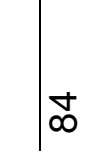 & 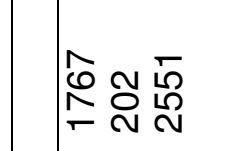 & 㐱 & $\stackrel{?}{?}$ & 皇 & 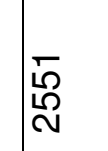 & 怘 \\
\hline 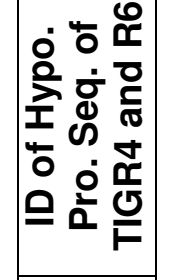 & 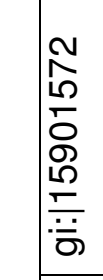 & 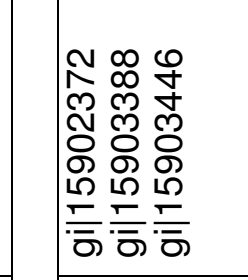 & 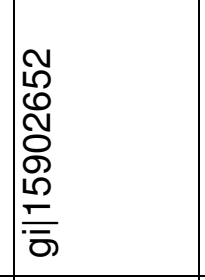 & $\begin{array}{l}\bar{\infty} \\
\stackrel{0}{0} \\
\delta \\
o \\
\stackrel{0}{\bar{\sigma}} \\
\overline{\bar{\sigma}}\end{array}$ & 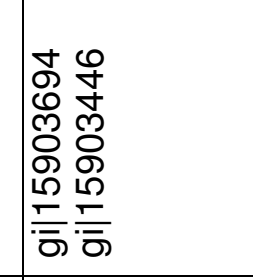 & 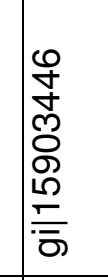 & 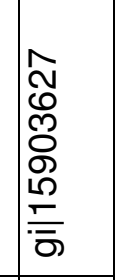 \\
\hline 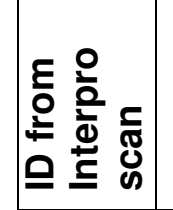 & $\begin{array}{l}8 \\
0 \\
0 \\
0 \\
0 \\
0\end{array}$ & 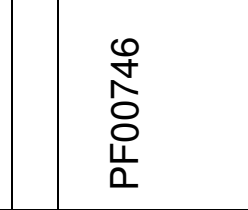 & 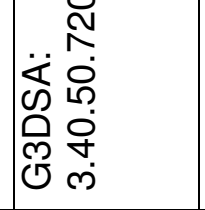 & 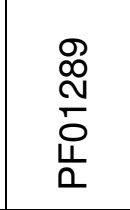 & $\begin{array}{l}0 \\
0.0 \\
0 \\
0 \\
0 \\
0 \\
0\end{array}$ & $\begin{array}{l}\bar{c} \\
\text { 足 } \\
0 \\
0 \\
0\end{array}$ & $\begin{array}{l}8 \\
0 \\
0 \\
0 \\
14 \\
0\end{array}$ \\
\hline
\end{tabular}


coccal surface protein A, autolysin, hyaluronate lyase, pneumolysin, neuraminidase B and pneumococcal surface antigen A of TIGR 4 are closely related to those of the other three strains, which shows that the virulence nature due to these factors among four strains seems to be similar. But the virulence factors neuraminidase $\mathrm{A}$, choline binding protein A and immunoglobulin A1 protease of TIGR4 differs from other strains of $S$. pneumoniae, which shows that these factors are responsible for the differences in virulence nature among four strains.

From the gene role category comparison, many genes of TIGR4 that are nearly same as in G54 and R6, suggests the basic complement of proteins required for certain cellular processes in the strains of $S$. pneumoniae. But many of the genes of TIGR4 which are notably different from the strains G54 and R6, suggest that these proteins are important for strain uniqueness and they may be involved in variations in pathogenesis. Since many hypothetical, conserved hypothetical, unknown and unclassified proteins exist among the dissimilar role categorized genes, it seems that many of these genes of $S$. pneumoniae have to be annotated and assigned functions of which some of them may also be responsible for the virulence nature. Further, we have also found that most of the virulence factors are same in D39 and R6 and hence also confirms the fact that R6 is the derivative of the strain D39.

In order to annotate the uncharacterized protein sequences (hypothetical and conserved hypothetical), the present study predicted 4 and 22 hypothetical sequences of the strains TIGR4 and R6 respectively of $S$. pneumoniae are of virulence factors. Among those predicted virulence factors, 1 and 8 different hypothetical sequences of TIGR4 and R6 respectively contain conserved sequences of known virulence factors such as hyaluronidase, immunoglobulin A1 protease, capsular polysaccharide synthesis, pneumolysin, neuraminidase and choline binding protein. These sequences also may be considered as desirable targets for therapeutics. The effort is to narrow down the search of virulence factors from all hypothetical sequences and this conclusion will be a reality only when it is experimentally proved.

\section{References}

1. AlonsoDeVelasco E, Verheul AF, Verhoef J, Snippe H (1995) Streptococcus pneumoniae: virulence factors, pathogenesis, and vaccines. Microbiol Rev 59: 591-603. "CrossRef " Pubmed " Google Scholar

2. Avery OT, MacLeod CM, McCarty M (1979) Studies on the chemical nature of the substance inducing transformation of pneumococcal types. Inductions of trans- formation by a deoxyribonucleic acid fraction isolated from pneumococcus type III. J Exp Med 149: 297-326. "CrossRef " Pubmed " Google Scholar

3. Bork P (2000) Powers and pitfalls in sequence analysis: the 70\% hurdle. Genome Res 10: 398-400. "CrossRef " Pubmed " Google Scholar

4. Brown TA Jr, Ahn SJ, Frank RN, Chen YY, et al. (2005) A hypothetical protein of Streptococcus mutans is critical for biofilm formation. Infect Immun 73: 3147-3151.» CrossRef " Pubmed " Google Scholar

5. Brückner R, Nuhn M, Reichmann P, Weber B, Hakenbeck R (2004) Mosaic genes and mosaic chromosomes - genomic variation in Streptococcus pneumoniae. Int J Med Microbiol 294: 157-168. »CrossRef " Pubmed " Google Scholar

6. Dopazo J, Mendoza A, Herrero J, Caldara F, Humbert Y, et al. (2001) Annotated draft genomic sequence from a Streptococcus pneumoniae type 19F clinical isolate. Microb Drug Resist 7: 99-125. » CrossRef » Pubmed » Google Scholar

7. Dowson CG (2004) What is a Pneumococcus? In Tuomanen et al. (eds)The Pneumococcus ASM press Washington pp 3-14.

8. Ferretti JJ, Ajdic D, McShan WM (2004) Comparative genomics of streptococcal species. Indian J Med Res 119: 1-6.»CrossRef » Pubmed » Google Scholar

9. Galperin MY, Koonin EV (2004) Conserved hypothetical proteins: prioritization of targets for experimental study. Nucleic Acids Res 32: 5452-5463. " "CrossRef » Pubmed » Google Scholar

10. Garg A, Gupta D (2008) VirulentPred: a SVM based prediction method for virulent proteins in bacterial pathogens. BMC Bioinformatics 28: 9-62. "CrossRef » Pubmed " Google Scholar

11. Gregory TR, DeSalle R (2005) Comparative genomics in prokaryotes. In Gregory (ed.) The evolution of the genome, Elsevier/Academic Press. London pp 585-660.

12. Hoskins J, Alborn WE Jr, Arnold J, Blaszczak LC, et al. (2001) Genome of the bacterium Streptococcus pneumoniae strain R6. J Bacteriol 183: 5709-5717. "CrossRef » Pubmed » Google Scholar

13. Hughes D (2000) Evaluating genome dynamics: the constraints on rearrangements within bacterial genomes. Genome Biol 1: reviews 0006.1-0006.8. »CrossRef » Pubmed " Google Scholar

14. Jedrzejas MJ (2001) Pneumococcal virulence factors: structure and function. Microbiol Mol Biol Rev 65: 187 207. "CrossRef » Pubmed " Google Scholar

15. Jothi R, Manikandakumar K, Ganesan K, Parthasarathy S (2007) On the analysis of the virulence nature of TIGR4 
and R6 strains of Streptococcus pneumoniae using genome comparison tools. J Chem Sci 119: 559-563. »CrossRef » Google Scholar

16. Lanie JA, Wai LNG, Kazmierczak KM, Andrzejewski TM, Davidsen TM, et al. (2007) Genome sequence of Avery's virulent serotype 2 strain D39 of Streptococcus pneumoniae and comparison with that of unencapsulated laboratory strain R6. J Bacteriol 189: 38-51. "CrossRef " Pubmed " Google Scholar

17. Polissi A, Pontiggia A, Feger G, Altieri M, Mottl H, et al. (1998) Large-scale identification of virulence genes from Streptococcus pneumoniae. Infect Immun 66: 56205629.»CrossRef » Pubmed » Google Scholar

18. Rigden DJ, Galperin MY, Jedrzejas MJ (2003) Analysis of structure and function of putative surface-exposed proteins encoded in the Streptococcus pneumoniae genome: A Bioinformatics-based approach to vaccine and drug design. Crit Rev Biochem Mol Biol 38: 143-168. " Pubmed " Google Scholar

19. Silva NA, McCluskey J, Jefferies JM, Hinds J, Smith A, et al. (2006) Genomic diversity between strains of the same serotype and multilocus sequence type among pneumococcal clinical isolates. Infect Immun 74: 3513-

3518. "CrossRef » Pubmed » Google Scholar

20. Sivashankari S, Shanmughavel P (2006) Functional annotation of hypothetical proteins - A review. Bioinformation 1:335-338. »CrossRef » Pubmed » Google Scholar

21. Tettelin H, Hollingshead SK (2004) Comparative genomics of Streptococcus pneumoniae: Intrastrain diversity and genome plasticity. In Tuomanen etal. (eds) The Pneumococcus ASM press Washington pp 15-29. »CrossRef » Google Scholar

22. Tettelin H, Nelson KE, Paulsen IT, Eisen JA, Read TD, et al. (2001) Complete genome sequence of a virulent isolate of Streptococcus pneumoniae. Science 293: 498506. "CrossRef » Pubmed » Google Scholar

23. Wizemann TM, Heinrichs JH, Adamou JE, Erwin AL, Kunsch C, et al. (2001) Use of a whole genome approach to identify vaccine molecules affording protection against Streptococcus pneumoniae infection. Infect Immun 69: 1593-1598. »CrossRef » Pubmed » Google Scholar 